\title{
Results of 5-year photometric monitoring of the intermediate polar V2306 Cygni: correction of the orbital period and evidence of 2-day periodicity
}

\author{
V. Breus ${ }^{1 *}$ K. Petrík ${ }^{2}, S . Z_{0 ł a}^{3,4}$, A. Baransky ${ }^{5}$, T. Hegedus ${ }^{6}$ \\ ${ }^{1}$ Department of High and Applied Mathematics, Odesa National Maritime University, Odesa, Ukraine \\ ${ }^{2}$ Hlohovec Astronomical Observatory, Hlohovec, Slovak Republic \\ ${ }^{3}$ Astronomical Observatory of the Jagiellonian University, Krakow, Poland \\ ${ }^{4}$ Mt. Suhora Observatory, Pedagogical University, Krakow, Poland \\ ${ }^{5}$ Astronomical Observatory, Taras Shevchenko National University of Kyiv, Kyiv, Ukraine \\ ${ }^{6}$ Baja Astronomical Observatory, Baja, Hungary
}

\begin{abstract}
We present the results of 6 years of photometric monitoring of the magnetic cataclysmic variable V2306 Cygni (formerly known as 1WGA J1958.2+3232) obtained at collaborating observatories. Using (O-C) analysis we tried to study variability of the spin period of the white dwarf, however we cannot make a firm conclusion based on the scatter. Simultaneously, using (O-C) diagram of orbital minima, we found that the value of $0.181545(3)$ days better corresponds with the light curve, than do previously published orbital period values. We also found that the variability has a 2.01 day period; this variability may be interpreted as possible precession of the accretion disk in this system.
\end{abstract}

Key words: stars: binaries: close - stars: novae, cataclysmic variables - stars: individual (V2306 Cygni) - stars: binaries: close - accretion disks - novae, cataclysmic variables

\section{INTRODUCTION}

The pulsating X-ray source 1WGAJ1958.2+3232 was discovered by Israel et al. [4]. Authors discussed the possible nature of the 12-min pulsations and concluded that the source may be a long-period, lowluminosity X-ray pulsar, or an intermediate polar. Later on, Negueruela et al. [7] classified this object as an intermediate polar by its X-ray and optical characteristics. They noticed the double-peaked structure of the emission lines that indicates the presence of the accretion disc.

Uslenghi et al. [9] suggested that the true spin period of the white dwarf could be 24 min rather than $12 \mathrm{~min}$, and mentioned that this object has one of the slowest rotators exhibiting a double-peaked spin profile.

Zharikov et al. [10] reported the detection of the orbital period of $4 \mathrm{~h} 36 \mathrm{~m}\left(0^{d} .1802 \pm 0^{d} .0065\right)$ from photometry and the final value of $0^{d} .18152 \pm 0^{d} .00011$ from radial velocity variations. They confirmed the $(733.82 \pm 1.25)$ s. spin period of the white dwarf using the spectroscopy and photometry and interpreted strong modulations with orbital period in the emission lines as a presence of a bright hot spot on the edge of the accretion disk.

\footnotetext{
*Vitaly.Breus@gmail.com

(C) V.Breus, K. Petrík, S.Zoła, A. Baransky, T. Hegedus, 2015
}

Later on, Norton et al. [8] reported that the orbital period is $(5.387 \pm 0.006)$ hours, corresponding to the $\sim 1$ day alias of the period found by Zharikov et al. [10] and confirmed that the rotational period of the white dwarf is twice the pulse period.

Soon afterwards, Zharikov et al. [11] repeated the analysis using own data along with the data provided by A. Norton. They confirmed their previous results and published the final value of $0^{d} .181195 \pm$ $0^{d} .000339$. However, they mentioned that a longer time base of observations is needed to improve this value.

The star was named as V2306 Cyg in 2003. In this paper we present the results of the long-term multicolour photometric CCD monitoring of this system.

\section{OBSERVATIONS}

The CCD photometric observations of the V2306 Cyg were obtained using 60-cm ZeissCassegrain telescope at the Observatory and Planetarium in Hlohovec, Slovakia (ZC600) equipped with SBIG ST-9 camera, 50-cm Zeiss reflector at the Fort Skala Observatory in Krakow, Poland (Zeiss50) equipped with Andor DZ936 camera, 70-cm AZT8 telescope at the Astronomical Observatory of the Taras Shevchenko National University of Kyiv, 
Ukraine (AZT-8) equipped with PL47-10 FLI and 50-cm reflector of Baja Astronomical Observatory, Hungary $(50 \mathrm{~cm})$ using Apogee camera. Along with our time series we analyzed all long CCD time series published in the AAVSO database (14 runs). The observations log is presented in Table 1 . The length of the observational run is given in hours. HJD is given with subtracted 2450000.0. The telescope column shows the telescope name from listed above, or 3-4 letter AAVSO observer code.

The CCD frames were processed using C-MuniPack software package. The final time series were obtained using the program MCV [2] taking into account multiple comparison stars method [5], the same software was used for periodogram analysis.

To determine spin maxima and orbital minima timings we used trigonometric polynomial approximation. We chose a 2-periodic variability model for smoothing:

$m(t)=m_{0}-r_{1} \cos \left[\omega_{1}\left(t-T_{01}\right)\right]-r_{2} \cos \left[\omega_{2}\left(t-T_{02}\right)\right]$,

where $m(t)$ is the smoothed value of brightness at time $t, m_{0}$ is average brightness on theoretical curve (generally different from the sample mean [1], $\omega_{j}=$ $2 \pi / P_{j}, r_{j}$ is semi-amplitude, $T_{0 j}$ is the epoch for maxima of brightness of photometric wave with number $j$ and period $P_{j}$. We calculated only one moment per set of observations (i. e., per night) because the accuracy estimate is much better then for individual extrema. This method is regularly used for approximation of observations of intermediate polars, e.g., MU Cam [6], EX Hya [3].

We took into account only best 41 spin and 41 orbital timings from 46 determined using all photometric data. To study period variations we used (O-C) analysis. Two O-C diagrams were built: for spin maxima and for orbital minima timings.

Contrary to a classical representation of the "O$\mathrm{C}$ diagram" as a dependence of the timings from an ephemeris, i.e.,

$$
O-C=T-\left(T_{0}+P \cdot E\right)
$$

on the cycle number $E$ we have used phases instead, i. e. $\phi=(O-C) / P$. For a correct ephemeris, the phases should be concentrated near the zero value.

\section{VARIABILITY OF THE \\ WHITE DWARF SPIN PERIOD}

Period variations are frequently observed in intermediate polars and are typically detectable at a time scale of decades. Using polynomial fits to the (O-C) diagrams it is possible to study variations of the spin period of the white dwarf. Using coefficients of these approximations after many years of monitoring, it is possible to determine the value of the period more precisely, find period variability and (in some cases) detect second derivatives of the period, e.g. acceleration of the spin period of the white dwarf. During our research in some objects we found a spin-up, sometimes the period increase was turned to a period decrease, sometimes the period is constant.

We tried to study variations of the spin period of V2306 Cygni the same way. Unfortunately, the relatively short timescale and large scatter at the (O-C) makes all attempts to correct cycle miscount ambiguous. Longer time base is needed, but we found no published spin maxima timings or time series obtained earlier.

\section{ON THE ORBITAL PERIOD}

We used the preliminary value of the orbital period of $0^{d} .22446[8]$ among two published aliases of each other for (O-C) analysis due to larger amplitude of variations at the phase curves for some selected nights of observations. We found regular cycle miscount, and minima timings obtained during different years looked like separate trends not connected to each other. Using consecutive photometric runs obtained in May-June 2014 (see Figure 1) we got the true cycle miscount and corrected it for all (O-C) data. After this, we smoothed the (O-C) diagram with a polynomial of statistically-optimal degree, which ended up being a linear approximation (see Figure 2). Using coefficients of the polynomial, we determined a more accurate value for the orbital period of V2306 Cyg, which was 0.2232685(24) days.

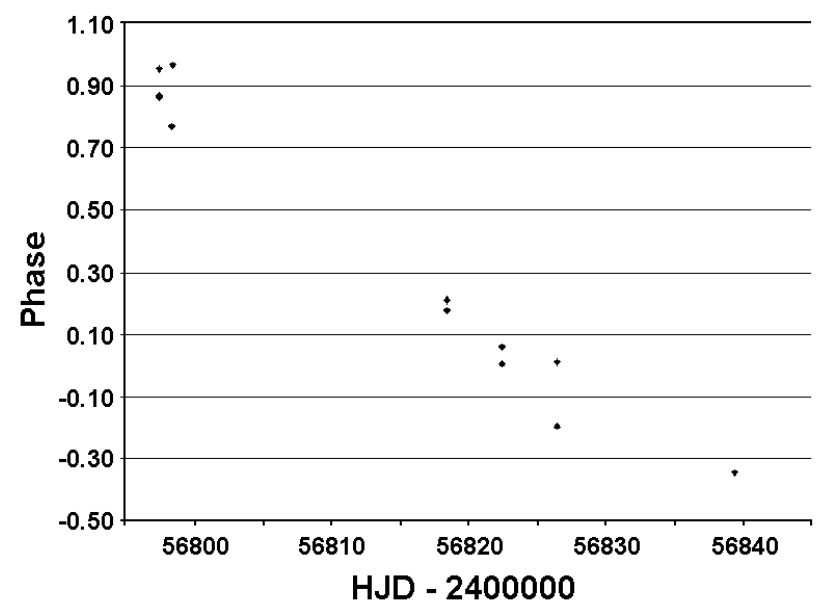

Fig. 1: (O-C) diagram of V2306 Cyg for orbital minima using data obtained in May-June 2014

Then we checked the (O-C) diagram for the period of $0^{d} .1825179$, which is a daily alias of our "corrected" value and close to that published by Zharikov et al. [10]. There were same separate linear trends from year to year. Using similar calculations we determined the new value of the orbital period of $0^{d} .181545 \pm 0^{d} .000003$ which is slightly different from the value of $0^{d} .181195 \pm 0^{d} .000339$ determined from 
radial velocities by Zharikov et al. [11].

Of course, longer time series and a larger time base enables to determine the orbital period of the system with better accuracy.

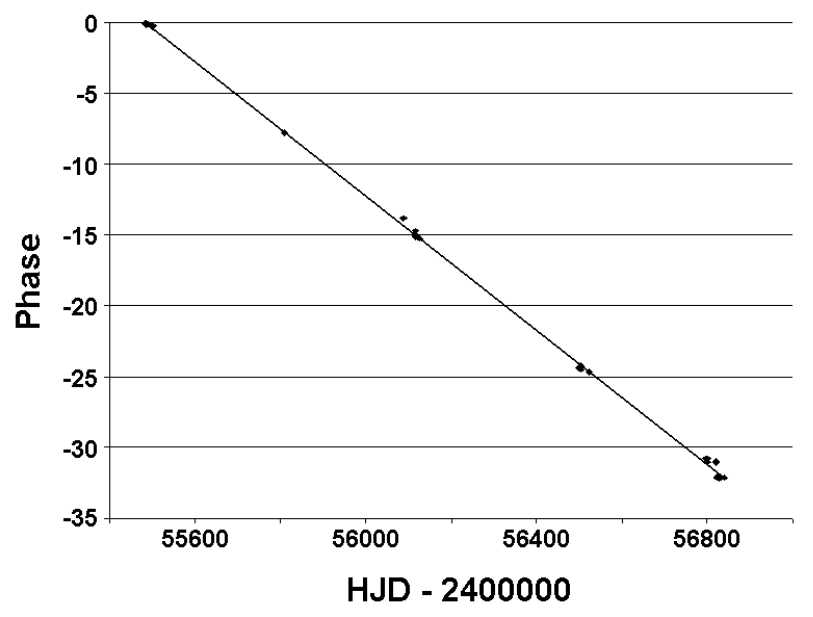

Fig. 2: (O-C) diagram of V2306 Cyg for orbital minima using data obtained in 2010-2014

\section{TWO-DAY PERIOD}

Periodogram analysis for the entire available time series revealed a prominent peak, which corresponds to the double value of the beat period of the orbital one and previously unknown one $\left(P=2^{d} .0183\right)$. This new period is visible at the larger range periodogram. Despite the fact that this value is a multiple of the length of the day and observations cover only part of the phase curve, we noticed significant ascending and descending branches of it due to different mean brightness from night to night (see Fig. 3). It is worth noting that the phase curve in $\mathrm{V}$ has a much larger amplitude than in $\mathrm{R}$ filter, so if this variability is real, it may be interpreted as a possible precession of the accretion disk in this system.

\section{RESULTS AND CONCLUSIONS}

We tried to find variability of the white dwarf spin period in the intermediate polar V2306 Cyg on the timescale of 5 years (2009-2014). Unfortunately, we found no unambiguous correction of present cycle miscount. However, using (O-C) analysis we corrected the value of the orbital period of this binary system and obtained the result $0^{d} .181545 \pm$ $0^{d} .000003$ which is consistent with the value published by Zharikov et al. [11]. Orbital phase curves of V2306 Cyg calculated with this period show larger amplitudes than all previously published or obtained by us for most seasons. Currently the accuracy estimate of our result is 113 times better. We report the detection of the $P=2^{d} .0183$ periodicity, which may be interpreted as the precession of the accretion disk in this system.

\section{ACKNOWLEDGEMENT}

We would like to thank William Gof, Etienne Morelle, James Jones, Richard Sabo, J. Ulowetz and the administration of the AAVSO International Database contributed by observers worldwide for the observations used in this research.

\section{REFERENCES}

[1] Andronov I. L. 2003, ASP Conf. Ser., 292, 391

[2] Andronov I. L. \& Baklanov A. V. 2004, Visnyk Astronomichnoi Shkoly, 5, 264

[3] Andronov I. L. \& Breus V. V. 2013, Astrophysics, 56, 518

[4] Israel G. L., Angelini L., Campana S. et al. 1998, MNRAS, 298, 502

[5] Kim Y., Andronov I. L. \& Jeon Y.-B. 2004, J. Astron. Space Sci., 21, 191

[6] Kim Y.-G., Andronov I. L., Park S.-S. et al. 2005, J. Astron. Space Sci., 22, 197

[7] Negueruela I., Reig P. \& Clark J. S. 2000, A\&A, 354, L29

[8] Norton A. J., Quaintrell H., Katajainen S. et al. 2002, A\&A, 384, 195

[9] Uslenghi M., Bergamini P., Catalano S., Tommasi L. \& Treves A. 2000, A\&A, 359, 639

[10] Zharikov S. V., Tovmassian G. H., Echevarría J. \& Cárdenas A. A. 2001, A\&A, 366, 834

[11] Zharikov S. V., Tovmassian G. H., Echevarría J. 2002, A\&A, 390, L23 
Table 1: The observations log

\begin{tabular}{cccccccccc}
\hline Date & HJD start & Length & Filter & Telescope & Date & HJD start & Length & Filter & Telescope \\
\hline 08.08 .2009 & 5052.3750 & 5.50 & V, R & $50 \mathrm{~cm}$ & 04.09 .2011 & 5808.6818 & 7.06 & CV & SRIC \\
16.08 .2009 & 5060.2994 & 7.79 & V & $50 \mathrm{~cm}$ & 05.09 .2011 & 5809.6591 & 6.81 & CV & SRIC \\
21.08 .2009 & 5065.3480 & 6.46 & V, R & $50 \mathrm{~cm}$ & 08.06 .2012 & 6086.6755 & 4.83 & CV & UJHA \\
26.08 .2009 & 5070.3032 & 7.80 & V, R & $50 \mathrm{~cm}$ & 08.07 .2012 & 6116.5184 & 1.62 & V, R & ZC600 \\
14.10 .2010 & 5483.6355 & 2.11 & CV & GFB & 08.07 .2012 & 6117.3566 & 5.44 & V, R & ZC600 \\
14.10 .2010 & 5484.2885 & 3.48 & CV & MEV & 16.07 .2012 & 6125.3642 & 4.48 & V, R & ZC600 \\
15.10 .2010 & 5484.6344 & 2.07 & CV & GFB & 27.07 .2013 & 6501.3376 & 4.27 & V, R & ZC600 \\
15.10 .2010 & 5485.2551 & 1.91 & CV & MEV & 28.07 .2013 & 6502.3183 & 4.78 & V, R & ZC600 \\
16.10 .2010 & 5485.6194 & 2.06 & CV & GFB & 19.08 .2013 & 6524.3219 & 3.36 & B, V, R & AZT-8 \\
18.10 .2010 & 5487.6122 & 3.42 & CV & JJI & 19.05 .2014 & 6797.3526 & 5.72 & V, R & Zeiss50 \\
20.10 .2010 & 5489.6194 & 2.09 & CV & GFB & 20.05 .2014 & 6798.3538 & 2.07 & V, R & Zeiss50 \\
21.10 .2010 & 5490.6186 & 1.67 & CV & GFB & 09.06 .2014 & 6818.3482 & 5.39 & V, R & ZC600 \\
27.10 .2010 & 5496.6146 & 2.06 & CV & GFB & 13.06 .2014 & 6822.3542 & 4.78 & V, R & ZC600 \\
01.11 .2010 & 5501.5970 & 2.09 & CV & GFB & 17.06 .2014 & 6826.3506 & 5.52 & V, R & ZC600 \\
02.11 .2010 & 5502.6016 & 2.06 & CV & GFB & 30.06 .2014 & 6839.4021 & 1.27 & V, R & ZC600 \\
\hline
\end{tabular}

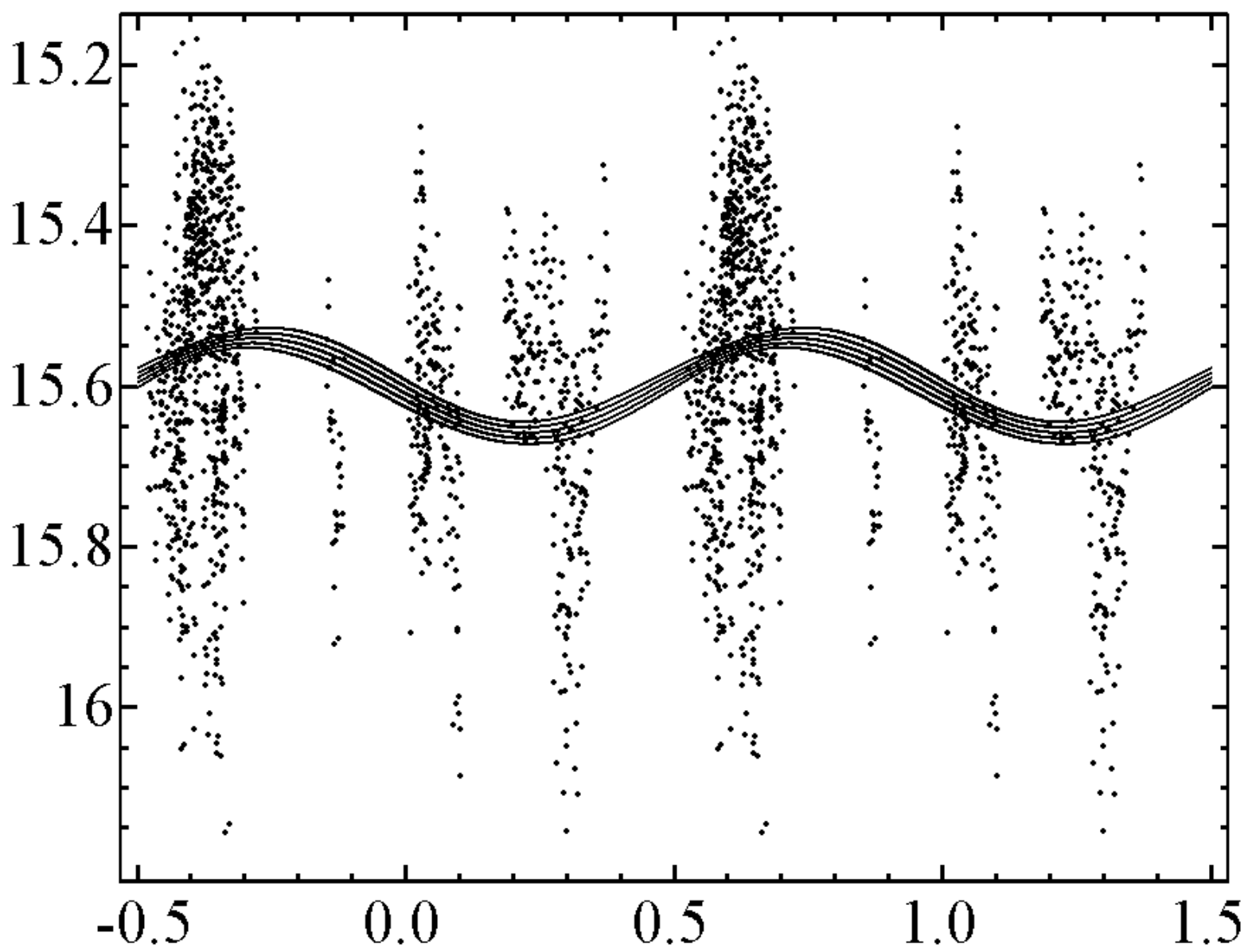

Fig. 3: Phase curve of V2306 Cyg for the period $P=2^{d} .0183$ using data obtained in Hlohovec in 2013 and 2014 . 